\title{
Pietraß, Manuela
}

\section{Was ist das Neue an "digitaler Bildung"? Zum hochschuldidaktischen Potenzial der elektronischen Medien}

Erziehungswissenschaft 28 (2017) 55, S. 19-27

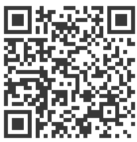

Quellenangabe/ Reference:

Pietraß, Manuela: Was ist das Neue an "digitaler Bildung"? Zum hochschuldidaktischen Potenzial der elektronischen Medien - In: Erziehungswissenschaft 28 (2017) 55, S. 19-27 - URN:

urn:nbn:de:0111-pedocs-152168 - DOI: 10.25656/01:15216

https://nbn-resolving.org/urn:nbn:de:0111-pedocs-152168

https://doi.org/10.25656/01:15216

in Kooperation mit / in cooperation with:

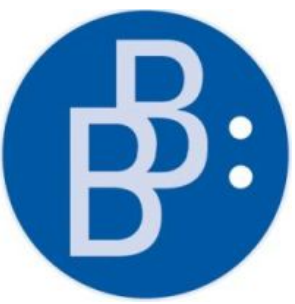

https://www.budrich.de

\section{Nutzungsbedingungen}

Dieses Dokument steht unter folgender Creative Commons-Lizenz: http://creativecommons.org/licenses/by-nd/4.0/deed.de - Sie dürfen das Werk bzw. den Inhalt vervielfältigen, verbreiten und öffentlich zugänglich machen, solange Sie den Namen des Autors/Rechteinhabers in der von inm festgelegten Weise nennen und das Werk bzw. diesen Inhalt nicht bearbeiten, abwandeln oder in anderer Weise verändern.

Mit der Verwendung dieses Dokuments erkennen Sie die

Nutzungsbedingungen an.

\section{Terms of use}

This document is published under following Creative Commons-License: http//creativecommons.org/licenses/by-nd/4.0/deed.en - You may copy, distribute and transmit, adapt or exhibit the work in the public as long as you attribute the work in the manner specified by the author or licensor. You are not allowed to alter or transform this work or its contents at all.

By using this particular document, you accept the above-stated conditions of use.

\section{(c) $(1)$}

\section{Kontakt / Contact:}

peDOCS

DIPF | Leibniz-Institut für Bildungsforschung und Bildungsinformation Informationszentrum (IZ) Bildung

E-Mail: pedocs@dipf.de

Internet: www.pedocs.de

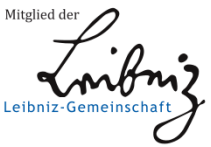




\section{Erziehungswissenschaft}

Mitteilungen der Deutschen Gesellschaft für Erziehungswissenschaft (DGfE)

Heft 55

28. Jahrgang 2017

ISSN 0938-5363

Verlag Barbara Budrich 


\section{INHALTSVERZEICHNIS}

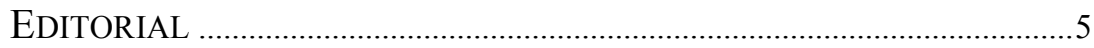

BEITRÄGE ZUM THEMA

„UNIVERSITÄT 4.0“. FOLGEN DER DIGITALISIERUNG

AKADEMISCHER LEHRE UND FORSCHUNG

Christian Swertz

Orientierungskönnen in der Leonardo-Welt

Manuela Pietraß

Was ist das Neue an „digitaler Bildung“? Zum hochschuldidaktischen

Potenzial der elektronischen Medien

Richard Stang

Lernraumgestaltung an Universitäten. Zur Relevanz physischer

Lernräume im Kontext der Digitalisierung...

Katharina Walgenbach

Elitebildung für alle? Massive Open Online Courses (MOOCs)

Ulf-Daniel Ehlers

Hochschulbildung digital. Abschied vom Ideal der Universitas?

Timo Hoyer \& Fabian Mundt

Personalisiertes Studieren, reflektiertes Lernen. Eine Analyse des

Studierverhaltens in digital gestützter Lehre.....

Ines Langemeyer \& Sabrina Schmid

Forschendes Lernen durch Mediengestaltung. Analysen zum

expansiven Lernen.

AllgEMEINE BEITRÄGE

Kirsten Puhr

Drei Thesen zu Forschungsbedingungen der Erziehungswissenschaft im Themenfeld Inklusion.

Eine Lektüre der Stellungnahme der Deutschen Gesellschaft für

Erziehungswissenschaft zu Inklusion (2017) .79 


\section{MitTEILUNGEN DES VORSTANDS}

Stellungnahme der Deutschen Gesellschaft für Erziehungswissenschaft (DGfE) zur Archivierung, Bereitstellung und Nachnutzung qualitativer Forschungsdaten in der Erziehungswissenschaft

Stellungnahme des DGfE-Vorstands zu den Reaktionen auf den

Beschluss, Hartmut von Hentig den Ernst-Christian-Trapp-Preis

abzuerkennen

\section{BERICHTE AUS DEN SEKTIONEN}

Sektion 1 - Historische Bildungsforschung......

Sektion 2 - Allgemeine Erziehungswissenschaft

Sektion 3 - Interkulturelle und International Vergleichende Erziehungswissenschaft (SIIVE)

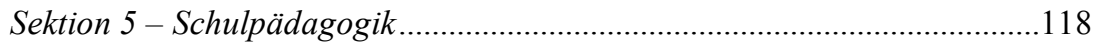

Sektion 8 - Sozialpädagogik und Pädagogik der frühen Kindheit................122

Sektion 9 - Erwachsenenbildung ............................................................130

Sektion 11 - Frauen- und Geschlechterforschung .......................................132

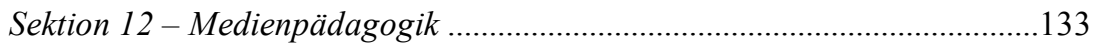

NOTIZEN

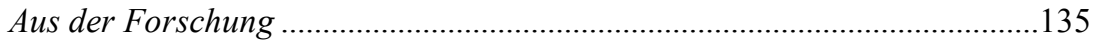

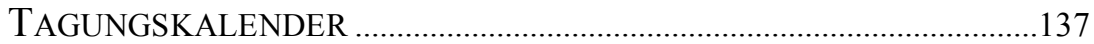

PERSONALIA

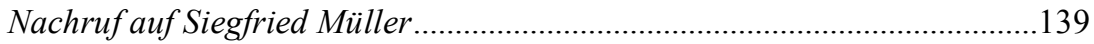




\title{
Was ist das Neue an ,digitaler Bildung"? \\ Zum hochschuldidaktischen Potenzial der elektronischen Medien
}

\author{
Manuela Pietraß
}

\section{Einleitung}

„Digitale Bildung“ ist derzeit in aller Munde. Selbstverständlich ist digitale Bildung die sprachlich verkürzte Form einer Bildung mit digitalen Medien. Auf dieses Neue verweist der Begriff und nicht auf eine - wie auch immer geartete - neue Form der Bildung an sich. Mit ihm wird auf einen Unterschied hingewiesen, der mit den digitalen Medien erzeugt wird, nämlich die mediale Verfasstheit von Angeboten, die für Bildung genutzt werden. Daran, dass die neue Technologie flächendeckend im Bildungssektor einzuführen und anzuwenden ist, wird kaum gezweifelt. Doch ist eine neue Technologie, die für Zwecke des Lehrens und Lernens eingesetzt wird, nicht per se eine Verbesserung. Vielmehr werden neue Medientechnologien anfangs meist so verwendet, wie die bereits bestehenden. Es wird also lediglich ,alter Wein in neue Schläuche gegossen“. Mancher angebliche Vorteil der digitalen Medien ruft deswegen aus medienpädagogisch-historischer Perspektive einen Déja-vu-Effekt hervor, so wie zu Beginn des E-Learning, als mit einem digitalisierten Handapparat und einem Chatforum aus Seminaren ,virtuelle Seminare“ wurden.

Was man mit jeweils neuen Medien anfangen kann, muss eine Gesellschaft immer erst herausfinden. Im Diskurs über digitale Bildung wird dementsprechend manches als neu angesehen, was es tatsächlich nicht ist, Anderes, was tatsächlich neu ist, wird noch kaum wahrgenommen. Möchte man aber das Bildungspotenzial der digitalen Medien verstehen, ist es wichtig, zwischen Altem im Neuen und Neuem im Neuen zu unterscheiden. Dies soll exemplifizierend am Strategiepapier des Bundesministeriums für Bildung und Forschung (BMBF) „Bildungsoffensive für die digitale Wissensgesellschaft“ (2016) vorgenommen werden, da es politischen Leitcharakter besitzt. Ausgehend von den dort der digitalen Bildung zugeschriebenen Charakteristika wird untersucht, inwiefern diese das Neue an digitaler Bildung erfassen oder bereits vorhandene Möglichkeiten strategisch steigern. Steigerung und Neuerung sind dabei nicht wertende, sondern analytische Kategorien, um das wesentlich Neue einer Bildung mit digitalen Medien freilegen zu können. Für die Durchsetzung und optimale didaktische Nutzung digitaler Medien ist deren Anwendung auf althergebrachte Weise, nur eben mit einem anderen Medium, nicht weniger wichtig. Doch entfalten diese Anwendungen ihr gesteigertes Potenzial vor dem Hinter- 
grund des Bisherigen und können vor diesem bewertet werden, während das Neue mit anderen Kategorien erfasst werden muss.

\section{Der Begriff der digitalen Bildung des BMBF}

Mit seiner „Bildungsoffensive für die digitale Wissensgesellschaft“ (2016) geht es dem BMBF grundsätzlich darum, die digitalen Medien auf breiter Ebene in allen Ausbildungsinstitutionen einzuführen. Digitale Bildung wird in zweierlei Hinsicht thematisiert: zum einen als „die Vermittlung digitaler Kompetenz“, was die Nutzung von digitalen Medien und mit ihnen angebotener Lehr- und Lerninhalte umfasst, zum anderen als ,das Lernen mit digitalen Medien (digitale Bildung als Instrument)“ (BMBF 2016, S. 8). „Digitale Kompetenz“" wird als Zieldimension digitaler Bildung verstanden und beinhaltet, nah am übergeordneten Begriff der Medienkompetenz angesiedelt, die Informationssuche, -bewertung und -produktion eigener Inhalte mit digitalen Medien, wobei die „Kompetenz zum selbsttätigen Lernen“ im Vordergrund steht. Weiterhin umfasst „,digitale Kompetenz“ explizit auch Medienkompetenz als „ein technisches Grundverständnis, das über die Bedienung aktueller Geräte hinausgeht und Grundkenntnisse über ihre Funktionsweise" einschließt (ebd.).

Neue Anwendungsmöglichkeiten des Lehrens und Lernens mit digitalen Medien sieht das BMBF in folgenden Punkten:

- flexibles, zeit- und ortsunabhängiges Lernen,

- größere Möglichkeiten der Interaktion zwischen Lehrenden und Lernenden, aber auch der Lernenden untereinander (kollaboratives Lernen),

- Anpassung der Inhalte digitaler Medien an die individuellen Bedürfnisse der Lernenden,

- schnelles Feedback und gezielte individuelle Förderung durch individualisierte, digital gestützte Lernformen und -strategie bei gemeinsamen Lernzielen,

- neue Erkenntnisse über Lehr- und Lernprozesse durch Erhebung und statistische Auswertung lehr- und lernbezogener Daten (BMBF 2016, S. 8).

Zusammengefasst ist aus der Sicht des BMBF digitale Bildung Bestandteil von Medienkompetenz, schließt die technische Nutzungsfähigkeit ein und zielt auf Selbstbestimmung und gesellschaftliche Teilhabe. Hinsichtlich des dafür erforderlichen Einsatzes digitaler Medien werden deren Nutzung für Bildung und Wirkung der Nutzung als Bildung gleichgesetzt. Offen bleibt also, auf welche Weise diese Anwendungsformen Bildung in einer Weise befördern, die bildungstheoretischen Ansprüchen genügt, oder ob Bildung lediglich als „Containerbegriff“ (Lenzen 1997, S. 949) verwendet wird mit Bezug auf das Bildungssystem insgesamt als organisationale Kategorie. 


\section{Medien dienen der Kommunikation - ein bildungsrelevanter Zusammenhang}

Um das didaktische Potenzial der digitalen Medien genauer bestimmen zu können, sollen die rein formal-technischen Nutzungsmöglichkeiten hinsichtlich dessen näher betrachtet werden, wozu sie dienen: Medien sind zunächst Mittel für Kommunikation. Mit Bildung sind Medien verknüpft, weil Lerngegenstände medial aufbereitet und vermittelt werden, und auch der Kontakt zwischen Lehrenden und Lernenden und Lernenden untereinander kann zunehmend medial vermittelt werden. Weil Medien Mittel für Kommunikation sind und Lehren durch Kommunikation erfolgt, liegt in der Kommunikation ein Ansatzpunkt, der Bildung und Medien theoretisch aufeinander beziehen lässt. Dieses Vorgehen ist nicht mit einer kommunikativen Pädagogik zu verwechseln, die sich mit der Frage befasst, wie sich die Auseinandersetzung zwischen Lehrenden und Lernenden kommunikationstheoretisch deuten lässt. Sondern es ist in der deiktischen Bedeutung von Kommunikation begründet, wie dies Prange (2012) mit der kommunikativen Operation des Artikulierens als didaktischem Gang des Zeigens darlegt.

Medien wohnt das Potenzial inne, Bildung dort zu realisieren, wo das Wissen der Lehrenden an seine Grenzen stößt, z.B. aufgrund eines beschränkten Erinnerungsvermögens, und wo Lehrkommunikation durch direkte Präsenz von Lehrperson und Lernenden eingeschränkt wird. Anders formuliert: Medien sind Instrumente, die eine Lehrkommunikation auch dann ermöglichen, wenn die Kommunikation zwischen Lehrperson und Lernenden unwahrscheinlich wird, weil mit Medien Kommunikation aus der Ferne vollzogen werden kann (Esposito 2006, S. 64). Erreichen können Medien dies, indem sie den für die Kommunikation notwendigen Zusammenhang zwischen Mitteilung und Information (Luhmann 2005) aufheben, um dann dennoch durch sich selbst Kommunikation zu ermöglichen. Dazu stellen sie Formen bereit, z. B. eine Lernsoftware oder eine virtuelle Erfahrungswelt wie eine Simulation. In diesen Formen werden neue Weisen erzeugt, Welt zu erfahren und zu verstehen: Erfahrung im erlebenden und Erkenntnis erzeugenden Vollzug, Verstehen im Sinne der Schaffung von Perspektiven auf Welt.

Die durch die neuen Medientechnologien gegebenen Möglichkeiten der Informationsvermittlung, beruhend auf der Trennung von Mitteilung und Information, führen dazu, dass geeignete Formen benötigt werden, um das jeweilige Vermittlungspotenzial von Medien darin zu realisieren. Hierbei ist festzustellen, dass historisch gesehen eine zeitliche Verschiebung auftritt, während der die neuen wie die vorangehenden Medien genutzt werden, weil das Potenzial für neue Anwendungsformen jeweils erst entdeckt und entwickelt werden muss. Hinsichtlich dieser Verschiebung werden die verschiedenen Entwicklungsstufen von Medien folgend dargestellt, um den Unterschied, den Digitalität konstituiert, erfassbar werden zu lassen. Dabei geht es 
darum, den Unterschied zwischen auf bisherigen und auf neuen Formen beruhenden Anwendungsformen zu exemplifizieren, wobei eine vertiefte Darstellung anhand der einschlägigen Werke zur Medienentwicklung (insbesondere McLuhan 1968; Giesecke 1985; Kittler 1995) hier nicht geleistet werden kann. Als mediengeschichtliche Phasen werden die Sprache, die Schrift, der Buchdruck, Film und Funk und der Computer unterschieden.

Bei der sprachlichen Kommunikation sind Mitteilung, Information und Verstehen direkt miteinander verknüpft: Der Mitteilende, der in einer sozialen Beziehung zu jenen steht, an die die Mitteilung gerichtet ist, formuliert das, was er mitteilen möchte, mit der Information. Dazu ist eine zeitliche und räumliche Anwesenheit von Mitteilendem und Verstehendem notwendig. Entsprechend sind orale Kulturen ,von der Flüchtigkeit der gesprochenen Rede und den durch die Mündlichkeit bestimmten Kommunikationsbedingungen“ geprägt (Schmidt 1998, S. 94). Tatsächlich beginnt bereits mit der Sprache die Trennung von Mitteilung und Information in der mit ihr gegebenen Möglichkeit, in Abwesenheit von einem Ereignis über dieses Ereignis zu sprechen. Dies führt zu dem Problem, wie man solche Ereignisse speichern könne.

Orale Kulturen müssen die intra- und intergenerationelle Weitergabe von Wissen bewältigen, wozu die Entwicklung der Schrift diente (Esposito 2006, S. 65). Sie erlaubte es, die dazu entwickelten Mnemotechniken in ihrer Funktionalität zu übertreffen. Dieser Bedeutung entsprechend diente das Medium Schrift ursprünglich der Gedächtnishilfe, während erst entdeckt werden musste, dass sie auch als Mittel zur Kommunikation dienlich sei, wie mit dem Briefwechsel (ebd.). Denn die Schrift ermöglichte es, Information so aufzubereiten, dass neue Formen der Kommunikation und mit ihr neue Sozialformen entstehen konnten, wie die Beurkundbarkeit von Sprache in Schrift. Hier ist wiederum ein Rest der Verbindung von Mitteilung und Information enthalten durch die Unterschrift, die von der Person in Anwesenheit geleistet werden muss, auf einem Schriftstück, welches die Loslösung der Beurkundung von der Person erst ermöglicht.

Eine ähnliche Verschiebung wie bei Einführung der Schrift wird wieder an der Entwicklung der Printkultur, die zunächst einmal nichts anderes bedeutete, als Schrift zu vervielfältigen, sichtbar, und der stark an handschriftliche Texte angelegten Form früher Drucke. Doch konnten durch die Vervielfältigbarkeit neue, in Textsorten gefasste Erkenntnisformen entwickelt werden, die prinzipiell mit der Schrift möglich gewesen wären, doch an ihrem Aufwand gescheitert sind. So kann als kategorial neu am Buchdruck gesehen werden, dass erst mit den beweglichen Lettern jene Wiederholbarkeit, Prüfbarkeit, Präzision der Beobachtung und Beschreibung sowie der Reflexivität des Schreibens erreichbar wurde, die für die Entwicklung der modernen Wissenschaften Voraussetzung war (Schmidt 1998, S. 98).

Nach der Drucktechnologie wurden Medien entwickelt, die Esposito (2006, S. 67) in Anlehnung an Luhmann als „Wahrnehmungsmedien“ bezeichnet. Sie 
beruhen auf Nachahmung und ermöglichen eine visuell-gegenständliche Wahrnehmung so, dass ihr Code als visuell und/oder auditiv getragene Wahrnehmung von Wirklichkeit fungiert. So waren für das Fernsehen als Wahrnehmungsmedium zunächst die Annäherung an die realitätsnahe Wahrnehmung und die Anlehnung an vorhandene Formate wie das Theater vorbildhaft. Erst in den 1990er Jahren gelang es dem Fernsehen, seine eigene Abbildhaftigkeit in sogenannten Hybridgenres und dem gleichzeitig entstehenden Eindruck einer wachsenden „Vielfalt von Medienwirklichkeiten“ (Pietraß 2003) zu thematisieren. Kategorial neu an den Wahrnehmungsmedien ist, dass die durch sie ermöglichte Trennung von Mitteilung und Information $\mathrm{zu}$ einer zunehmenden Unklarheit der Autorschaft einer Information, aber zugleich zu der direkten, prozesshaften Abbildbarkeit sozialer Wirklichkeit führt - es wird nicht über sie geschrieben, sondern sie wird in einem mimetischen Sinne neu geschaffen. Diese Nähe zu einer realitätsnahen Wahrnehmung führt dazu, dass das, was ausgesagt werden soll und das, was wahrgenommen wird, zu trennen sind.

Worin bei den digitalen Medien die Entdeckung neuer Anwendungen liegt, ist insofern schwierig endgültig zu bestimmen, als wir uns noch im Prozess dieser Entwicklung befinden. Grundsätzlich bestehen auch hier neue Formen von Informationsaufbereitung aufgrund der Loslösung von der Mitteilung, womit sich der nächste Abschnitt näher befassen wird.

\section{Steigerung und Neuerung der Anwendungsformen digitaler Medien für Bildung}

Folgend soll unter Bezug auf die oben dargestellte Bildungsoffensive des BMBF geprüft werden, inwiefern der dort verwendete Begriff einer digitalen Bildung auf bisherige bzw. auf neue Nutzungsweisen der digitalen Medien rekurriert, indem die oben zusammengefassten Punkte zum didaktischen Potenzial digitaler Bildung nochmals aufgenommen werden. Sie werden in zwei Abschnitte gegliedert: in Argumente, bei denen eine Verschiebung tradierter Anwendungsformen auf die neuen Medien sichtbar wird (1), und in Argumente, bei denen kategorial neue Aspekte aufgenommen werden (2).

\section{(1) Argumente des Strategiepapiers, die auf eine Steigerung bisheriger, tradierter Anwendungsformen zielen}

\section{Flexibles, zeit- und ortsunabhängiges Lernen}

Bücher waren durch den zunehmend kostengünstigeren Druck und auch durch die Einrichtung von Bibliotheken bereits einer großen Zahl Studierender zugänglich, aber mit Barrieren wie beschränkter Zahl an Exemplaren oder beschränkten Ausleihfristen und -zeiten. Hier bieten die digitalen Medi- 
en prinzipiell unbeschränkte Vervielfältigungs- und Speichermöglichkeiten sowie prinzipiell zeitlich unbegrenzte Zugangsmöglichkeiten. Die Neuerung gegenüber dem Buchdruck besteht in einer Steigerung bereits gegebener Möglichkeiten insofern, weil den Studierenden mehr Angebote zeitgleich und kostengünstiger zur Verfügung gestellt werden können, als dies mit gedruckten Büchern und Kopien in Handapparaten etc. der Fall war.

Eine weitere Steigerungsform gegenüber der Printkultur liegt in der verbesserten Standardisierungsmöglichkeit von Bildungsinhalten, sowohl hinsichtlich der Entwicklung von Formaten als auch der Angleichung und Verbreitung der Inhalte. Erleichtert wird dies durch die verbesserte Abstimmungsmöglichkeit der verschiedenen Bildungsanbieter untereinander sowie durch das digitale Kopieren und Bereitstellen von Texten auf speziellen Plattformen.

Anpassung der Inhalte digitaler Medien an die individuellen Bedürfnisse der Lernenden

Durch die erhöhte Angebotsauswahl von Inhalten findet eine Individualisierung beim Studieren statt: Bereits Bücher und auch die Wahrnehmungsmedien ermöglichten es, durch die Vielfalt der in beiden Kulturen entstandenen Formate und Genres, sich je nach Vorwissen und Vertiefungsinteresse eine individuelle Angebotsauswahl zusammenzustellen. Die erreichte Vielfalt kann mit digitalen Medien noch erhöht und zugleich das Angebot unaufwendig so kopiert werden, dass Inhalte und deren Darstellungsformen ausdifferenzierbar und die Verfügbarkeit von Wissensangeboten erhöht werden.

Auch Studienangebote werden individualisierbar durch eine erhöhte Angebotsauswahl: Eine weitere Steigerung gegenüber dem, was Medien prinzipiell bewirken - die Loslösung von Ort und Zeit - liegt in der neu erreichbaren Vielfältigkeit von Qualifizierungsangeboten, auch über die Lebensspanne hinweg, z. B. Fernstudiengänge, berufsbegleitendes Studieren und Open Educational Resources für informelles Lernen und virtuelle Qualifizierungsangebote für formales Lernen.

\section{(2) Argumente, die auf kategorial neue Anwendungsformen zielen}

Kategorial neue Anwendungsformen der digitalen Medien beruhen auf ihrer Interaktivität. Argumente, die sich darauf im Strategiepapier des BMBF (2016, S. 8) beziehen, sind folgende:

Interaktion zwischen Lehrenden und Lernenden und Lernenden untereinander, individualisierte, digital gestützte Lernformen, Erhebung und statistische Auswertung lehr- und lernbezogener Daten

Durch die Interaktivität digitaler Medien werden nicht nur bisherige Interaktionsformen verfeinert, sondern es besteht eine kategoriale Veränderung 
durch eine interaktive Herstellung von Texten mit Texten - wie die anfangs viel diskutierten Hyperlinks und das durch sie entstehende Text-,,Rhizom“ (Sandbothe 1997). Letztendlich sind alle Medien interaktiv im Sinne einer wechselseitigen Bezugnahme, welche Kommunikation voraussetzt (Manovich 2001). Eine direkte Wechselseitigkeit war jedoch bislang nur bei Funk und Telefonie möglich. Dies wird nun durch die digitalen Kommunikationsmöglichkeiten erweitert und eine wechselseitige Kommunikation realisierbar. Grundsätzlich verlangt auch die, die Trennung von Mitteilung und Information ermöglichende Kommunikation mit Medien den Einbezug des Verstehens, damit Kommunikation zustande kommt. Verstehen aber ist nur erreichbar, wenn der Mitteilende die Verstehensmöglichkeiten bereits antizipiert. Eine solche Antizipation ist bereits bei einem Gespräch erforderlich, aber bei den Medien ist diese, anders als bei einem Gespräch, von der Mitteilung gelöst. Dadurch erhält das „digitale Gespräch“ eine eigene, bearbeitbare Form, was besonders gut sichtbar wird bei kollaborativen, digital verschriftlichten Lernprozessen. Eine andere Form ist die Interaktion mit einem Text, der in sich Interaktionsmöglichkeiten eingeschrieben hat, z. B. durch Links oder durch Auswahloptionen von Antworten oder Handlungsmöglichkeiten. Durch die Interaktivität wird eine digitale „Spur“ erzeugt, welche einen eigenen Text darstellt, der im Prozess der Nutzung entsteht. Dadurch verändert sich - im Unterschied zur gesprochenen Kommunikation, nicht zum geschriebenen Text! - die Information. Sie wird nun, wie der geschriebene Text, strukturierbar, systematisierbar und speicherbar.

Das durch digitale Interaktivität erzeugte Kommunikat ermöglicht es durch seine Textförmigkeit, in eine reflexive Distanz zur Kommunikation zu treten. Dies ist besonders wichtig für die didaktische Besonderheit kollaborativen Lernens und auch für die Kommunikation zwischen Lernenden und Lehrenden, bei der auf den Lerninhalt bezogene Argumente ausgetauscht werden. Deren schriftliche Fixierung verlangt eine andere Form der Reflexion als die mündliche Äußerung und damit eine Metaperspektive auf den Lernprozess, doch zieht das zugleich Veränderungen nach sich, wie einen hohen Zeitaufwand und Zugangsbarrieren für jene, die sich mündlich sicherer äußern können. Einen ganz neuen Zugriff auf den Lernprozess, der in Form einer textuellen Lernspur abgebildet werden kann, erlauben digital gestützte Lernformen wie Portfolios. Deren Steigerung stellt der Zugriff auf die, durch die Textualität des Lernens ermöglichte, Datengewinnung und -speicherung dar.

\section{Zusammenfassung und Diskussion}

Auf Basis der Entwicklung von Formen der Informationsaufbereitung, was Medien durch die Trennung von Information und Mitteilung ermöglichen, werden die Steigerung bisheriger und die Entwicklung kategorial neuer Anwen- 
dungsformen digitaler Medien unterschieden: Als bisherige Anwendungsformen verstärkende Trends werden im Strategiepapier des BMBF thematisiert: der verbesserte Zugang zu universitärer Lehre und zur Weiterbildung sowie zu Bildungs- bzw. Wissensangeboten selbst, eine höhere Standardisierungsmöglichkeit von Lerninhalten und -prozessen mit einer zugleich bestehenden Individualisierung der Selektion des Lernangebotes. Diese Trends stellen eine Ausdifferenzierung und Ausweitung bereits in der Buchkultur gewachsener Möglichkeiten der Zugänglichkeit und der Verbreiterung des Angebotes dar.

Bei der didaktischen Analyse der Steigerung bisheriger Anwendungsformen sollten jene betrachtet werden, aus denen die Steigerung jeweils erfolgt. Dabei ist es nicht nur wichtig zu betrachten, welche bisherigen Begrenzungen nun überwunden werden können, sondern auch, inwiefern aus Fehlern und Erfolgen der früheren gelernt werden kann. Durch diesen Rückbezug ist eine Optimierung der vorhandenen in den neuen Anwendungsformen möglich.

Neue Trends liegen in der Interaktivität der digitalen Medien begründet. Sie bietet ein verändertes didaktisches Potenzial in der sozialen Kommunikation beim Lehren und Lernen, die schriftlich in den neuen Formen interaktiver Lernsoftware sowie in der Abbildung und Auswertung individueller Lernprozesse in Lernportfolios und anderen Formen der Lernprozessbeobachtung abbildbar wird. Bei dem auf textueller Interaktivität gestützten Lernen sind zwei Punkte didaktisch zu berücksichtigen: die Software bildet nicht „,den“ Lernprozess ab, sondern eine ganz bestimmte Form, und weiterhin, dass eine Verschiebung von der Individualität des Lernenden zu einem System stattfindet, das nur auf der Möglichkeit von Standardisierung seine Effektivität entfalten kann.

Vorliegend wurde am Bericht des BMBF exemplarisch durchgeführt, worin Argumente für das Neue digitaler Bildung bestehen, die auf frühere oder kategorial neue Anwendungsformen bezogen werden, ohne zu berücksichtigen, inwiefern der Bericht diesbezüglich vollständig ist. Grundsätzlich entfaltet sich dort, wo bisherige Anwendungsformen optimiert und nicht einfach nur fortgeführt werden und wo wesensmäßig andere als bisherige Anwendungsformen der digitalen gegenüber den bisherigen Medien angesiedelt sind, das neue didaktische Potenzial einer „digitalen Bildung“.

Manuela Pietra $\beta$, Prof. Dr., ist Hochschullehrerin für Erziehungswissenschaft mit Schwerpunkt Medienbildung an der Universität der Bundeswehr München.

\section{Literatur}

Bundesministerium für Bildung und Forschung (BMBF) (2016): Bildungsoffensive für die digitale Wissensgesellschaft. Strategie des Bundesministeriums für Bildung und Forschung. Berlin: Bundesministerium für Bildung und Forschung. 
Esposito, Elena (2006): Was man von den unsichtbaren Medien sehen kann. In: Soziale Systeme 12, 1, S. 54-78. https://doi.org/10.1515/sosys-2006-0105. Giesecke, Michael (1998): Der Buchdruck in der frühen Neuzeit. Eine historische Fallstudie über die Durchsetzung neuer Informations- und Kommunikationstechnologien. Frankfurt am Main: Suhrkamp.

Kittler, Friedrich (1985): Aufschreibesysteme 1800/1900. München: Fink.

Lenzen, Dieter (1997): Lösen die Begriffe Selbstorganisation, Autopoiesis und Emergenz den Bildungsbegriff ab? In: Zeitschrift für Pädagogik 43, 6, S. 949-968.

Luhmann, Niklas (2005): Einführung in die Theorie der Gesellschaft. Heidelberg: Carl-Auer.

Manovich, Lev (2001): The Language of the New Media. Cambridge, Massachusetts/London, England: Massachusetts Institute of Technology. http:// dss-edit.com/plu/Manovich-Lev_The_Language_of_the_New_Media.pdf [Zugriff: 5. Oktober 2017].

McLuhan, Marshall (1968): Die Gutenberg-Galaxis. Das Ende des Buchzeitalters. München: Econ.

Pietraß, Manuela (2003): Bild und Wirklichkeit. Zur Unterscheidung von Realität und Fiktion bei der Medienrezeption. Opladen: Leske + Budrich. https://doi.org/10.1007/978-3-322-93448-2.

Prange, Klaus (2012): Die Zeigestruktur der Erziehung. Grundriss der Operativen Pädagogik. 2. Auflage. Paderborn: Ferdinand Schöningh, S. 107-135.

Sandbothe, Mike (1997): Interaktivität - Hypertextualität - Transversalität. Eine medienphilosophische Analyse des Internets. In: Münker, S./Roesler, A. (Hrsg.): Mythos Internet. Frankfurt am Main: Suhrkamp, S. 56-82.

Schmidt, Siegfried J. (1998): Die Zähmung des Blicks. Konstruktivismus Empirie - Wissenschaft. Frankfurt am Main: Suhrkamp. 